\title{
Multimodal coherent anti-Stokes Raman scattering microscopy reveals microglia-associated myelin and axonal dysfunction in multiple sclerosis-like lesions in mice
}

\author{
Jaime Imitola, ${ }^{a, *}$ Daniel Côté, $,{ }^{b, d}, *$ Stine Rasmussen, ${ }^{a}$ X. Sunney Xie, ${ }^{b}$ Yingru Liu, ${ }^{a}$ Tanuja Chitnis, ${ }^{a}$ Richard L. Sidman, ${ }^{c}$ \\ Charles. P. Lin,,$\dagger$ and Samia J. Khoury ${ }^{a, \dagger}$ \\ a Brigham and Women's Hospital, Center for Neurologic Diseases, Partner Multiple Sclerosis Center, Harvard Medical \\ School, Department of Neurology, Boston, Massachusetts 02115 \\ ${ }^{b}$ Massachusetts General Hospital, Advanced Microscopy Program, Center for Systems Biology and Wellman Center for \\ Photomedicine, BHX 630, Harvard Medical School, 50 Blossom Street, Boston, Massachusetts 02114 \\ 'Beth Israel Deaconess Medical Center, Department of Neurology, Harvard Medical School, Boston, Massachusetts \\ 02115 \\ dUniversité Laval, Centre de Recherche Université Laval Robert Giffard and Centre d'Optique Photonique et Laser, \\ Québec City, Québec G1J 2G3, Canada
}

\begin{abstract}
Myelin loss and axonal degeneration predominate in many neurological disorders; however, methods to visualize them simultaneously in live tissue are unavailable. We describe a new imaging strategy combining video rate reflectance and fluorescence confocal imaging with coherent anti-Stokes Raman scattering (CARS) microscopy tuned to $\mathrm{CH}_{2}$ vibration of myelin lipids, applied in live tissue of animals with chronic experimental autoimmune encephalomyelitis (EAE). Our method allows monitoring over time of demyelination and neurodegeneration in brain slices with high spatial resolution and signal-to-noise ratio. Local areas of severe loss of lipid signal indicative of demyelination and loss of the reflectance signal from axons were seen in the corpus callosum and spinal cord of EAE animals. Even in myelinated areas of EAE mice, the intensity of myelin lipid signals is significantly reduced. Using heterozygous knock-in mice in which green fluorescent protein replaces the $\mathrm{CX}_{3} \mathrm{CR} 1$ coding sequence that labels central nervous system microglia, we find areas of activated microglia colocalized with areas of altered reflectance and CARS signals reflecting axonal injury and demyelination. Our data demonstrate the use of multimodal CARS microscopy for characterization of demyelinating and neurodegenerative pathology in a mouse model of multiple sclerosis, and further confirm the critical role of microglia in chronic inflammatory neurodegeneration. (C) 2011 Society of Photo-Optical Instrumentation Engineers (SPIE). [DOI: 10.1117/1.3533312]
\end{abstract}

Keywords: confocal; reflectance; coherent anti-Stokes Raman scattering; live imaging; $\mathrm{CX}_{3} \mathrm{CR} 1$-green fluorescence protein; microglia; neurodegeneration.

Paper 10157SSR received Mar. 24, 2010; revised manuscript received Sep. 15, 2010; accepted for publication Sep. 30, 2010; published online Feb. 17, 2011.

\section{Introduction}

Imaging methods for neurodegeneration are complicated by extensive manipulations of the tissue that may alter the expression of antigens. In addition, current methods to study demyelination require extensive tissue processing and are limited to histochemistry. Thus, new imaging methods that do not rely on labeling fixed tissue and can examine live samples would be valuable in the search for disease biomarkers. ${ }^{1}$ In recent years, several imaging techniques for the brain have been proposed, including two-photon microscopy in animals expressing fluorescent markers such as green fluorescent protein (GFP) in specific cellular compartments in the central nervous system (CNS), ${ }^{2,3}$ second harmonic generation microscopy to visualize membranes and

*These authors share first authorship.

†These authors contribute equally as co-senior authors.

Address all correspondence to: Samia J. Khoury, M.D., Brigham and Women's Hospital, Center for Neurologic Diseases, 77 Avenue Louis Pasteur, HIM 712, Boston, MA 02115, and Charles P. Lin, Massachusetts General Hospital, Advanced Microscopy Program, Harvard Medical School, 50 Blossom Street, Boston, MA 02114. Tel: 617-525-5370; Fax: 617-525-5501; E-mail: skhoury@rics.bwh.harvard.edu. their potential in vitro and in vivo, ${ }^{4,5}$ and coherent anti-Stokes Raman scattering (CARS) microscopy to study normal myelin in live and injured tissues. ${ }^{6,7}$ In addition, reflectance confocal microscopy (RCM) has been used to provide high-resolution optical sections of axons in the retinal nerve fiber layer ${ }^{8,9}$ and cellular changes in the skin. ${ }^{10}$ However, these techniques have not been tested to monitor the neurodegenerative process.

Experimental autoimmune encephalomyelitis (EAE) has been widely used as an animal model for multiple sclerosis (MS) and reproduces many of its neurodegenerative features. ${ }^{11}$ Neuropathological analysis has shown that the CNS inflammatory and demyelinating lesions in the mouse are typically located predominantly in the spinal cord, ${ }^{12}$ but we have recently shown that the forebrain is equally targeted in proteolipid protein (PLP)-induced EAE in mice of the SJL strain. ${ }^{13}$ The lesions are mostly perivenular in the cortical white matter. Periventricular areas and corpus callosum share several key features with MS, such as a preponderance of subpial lesions, and chronic activation of microglia. We have shown that in the forebrain,

1083-3668/2011/16(2)/021109/11/\$25.00 @ 2011 SPIE 
activation of microglia correlates with defects in axonal transport, axonal loss, and neuronal dysfunction. In the present paper, we have used a custom-built microscope that includes linear and nonlinear optical modalities to visualize live tissue ex vivo. This imaging method, termed multimodal CARS, combines for the first time three noninvasive imaging modalities for thick living tissue slices that are capable of visualizing axons with reflectance, ${ }^{8,9}$ GFP with fluorescence, and myelin with Raman in order to examine microglia-induced neurodegeneration. Our results indicate that these modalities can establish cellular correlates of MS-like lesions in mice and provide additional evidence for widespread neuronal and microglial dysfunction as the functional basis for disease progression in a mouse model of MS.

\section{Results}

\subsection{Custom-Built Multimodal Video Rate Microscope to Simultaneously Image Axons and Myelin in the Central Nervous System}

We custom constructed a microscope that allows simultaneous anatomical, chemical, and molecular imaging for the observation of axons, myelin sheaths, and immune cells. We achieve video-rate CARS imaging of live forebrain and spinal cord explants by selecting the frequency difference of the pump and Stokes beams so as to resonantly excite the stretching $\mathrm{CH}_{2}$ vibrations predominantly localized in lipids, thus allowing detection of CNS myelin. The CARS video-rate microscope has been described previously for study of lipid-rich cells in the skin of intact living mice and consists of two synchronized laser sources with a custom-built video-rate laser-scanning system. ${ }^{14,15}$ The microscope used in this study includes addition of a reflectance confocal channel, an improved filter stack before the nondescanned photomultiplier tube (PMT), an electronic limiter for analog thresholding of the CARS signal from the PMT, and a custom-built multichannel acquisition system with frameaveraging, photon-counting, and live-streaming capabilities. We used this microscope in four strains of mice [Figs. 1(a) and 1(b)] to obtain informative data on the dynamics of axons, myelin, and microglia in EAE.

\subsection{Simultaneous Imaging with Reflectance and Coherent Anti-Stokes Raman Scattering Microscopy Identifies Axons and Myelin in Normal Adult Forebrain}

To establish the ability of reflectance imaging to visualize CNS structures, first we used fixed CNS samples and examined specific brain sites [Fig. 2(a)], such as the corpus callosum and cortical layers II-VI. We detected an increase in the corpus callosum signal that delineated callosal projections between cerebral hemispheres [Fig. 2(b), left panel]. Next, we analyzed the reflectance signals in cortical layers II-VI. We could visualize axons projecting between corpus callosum and cortical layers [Fig. 2(b), right panel]. To determine the ability of reflectance in combination with CARS imaging to visualize live normal forebrain structures, we dissected slices of adult mouse forebrain and examined myelinated structures such as the corpus callosum and the cortex [Figs. 2(d) and 2(e)]. As a positive control for the CARS signals, we used tissue slices from mouse ears that were transferred to a coverslip and immersed in aqueous buffer to visualize individual sebaceous glands and

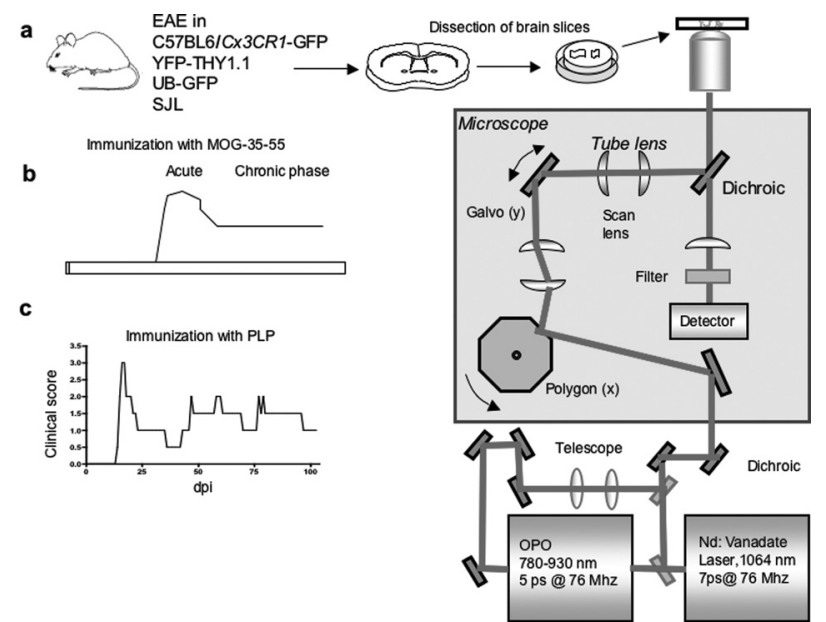

Fig. 1 Schematics of novel multimodal CARS microscopy to monitor inflammatory neurodegeneration. (a) Experimental schema of EAE induced in various strains. Tissue from spinal cord or forebrain was dissected and transferred to imaging slides of the multimodal CARS microscope. (b) Models of EAE used in the study, EAE in C57BL6 immunized with MOG 35-55 produces a chronic model of MS with an initial acute phase that leads to a chronic stable phase. (c) SJL strain develop a relapsing remitting disease when immunized with PLP 139 151 , which resembles a similar course that is observed in many MS patients.

adipocytes due to their high lipid content ${ }^{15}$ [Fig. 2(c)]. Individual sebaceous glands showed bright CARS signals and high contrast between the cytoplasm and nucleus of individual cells [Fig. 2(c), inset]. Using these parameters for image acquisition, we next evaluated the intensity of signals between heavily myelinated areas of the forebrain, such as the corpus callosum, and less myelinated areas, such as the cortex. We observed an increase in the CARS signal in the corpus callosum, in addition to individual myelin sheaths [Fig. 2(e) arrow]. Furthermore, we observed an increased intensity in the reflectance signal in the cortex compared to the corpus callosum [Fig. 2(e)], suggesting that CARS can visualize the signals from myelinated structures. To further confirm these observations, we analyzed the CARS signals from subcortical cerebellar white matter [Fig. 2(f)] of postnatal day 7 (that lack myelin), and cerebellum of seven-week-old mice (normally myelinated) and found increased CARS signal during developmental myelination [Figs. 2(f) and 2(g)]. These observations indicate that the combination of CARS and reflectance imaging can provide visualization of myelinated and unmyelinated axons in the normal adult and developing CNS.

\subsection{Coherent Anti-Stokes Raman Scattering Microscopy Provides Details Regarding the Substructures of Normal Central Nervous System Myelin}

We established that reflectance imaging can determine normal boundaries in the spinal cord between white matter (wm) and the gray matter $(\mathrm{gm})$. The reflectance signal is increased in the white matter because of the abundant numbers of axons, while anterior horn neuron cell bodies and dendrites have low reflectance [Fig. 3(a)']. This pattern is similar to that seen with Bielschowsky silver staining (data not shown). These observations suggest that 


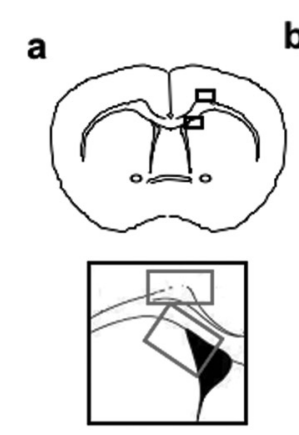

b

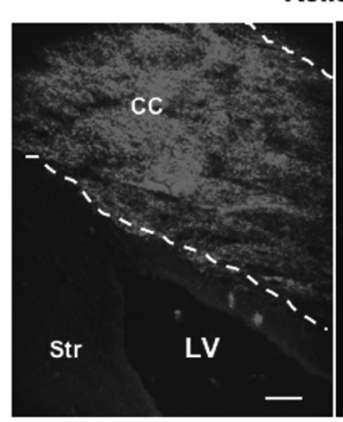

$\mathbf{e}$

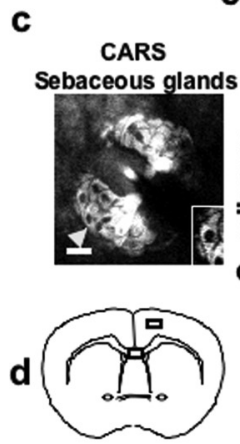

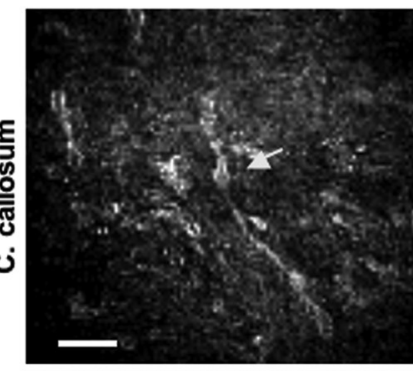

Reflectance

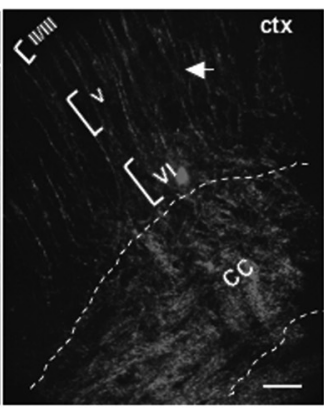

Reflectance

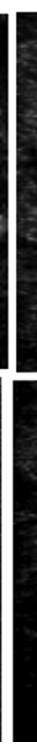

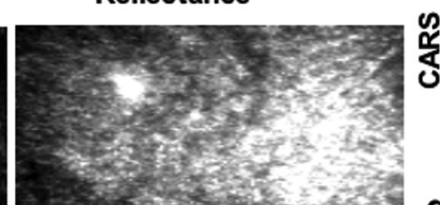

f

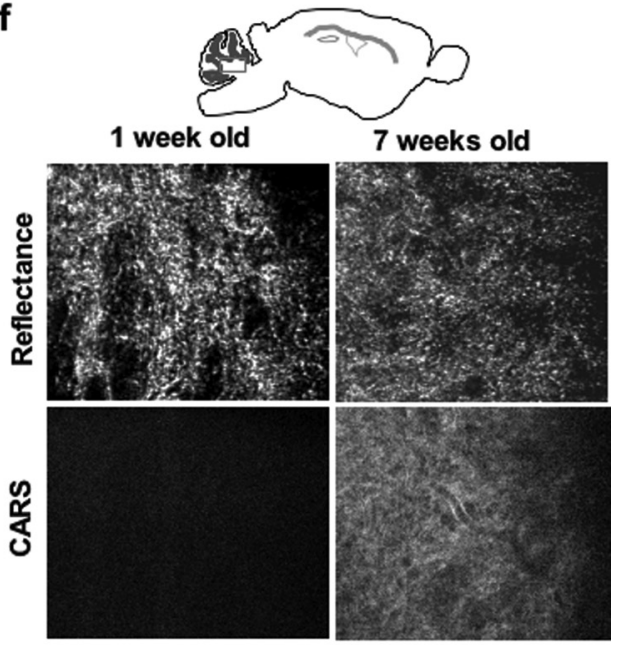

g
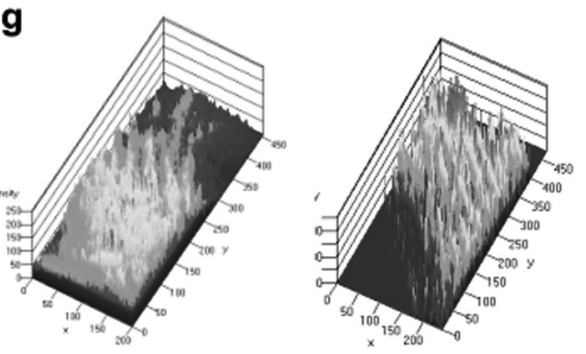

Fig. 2 Combination of CARS and reflectance demonstrate axons and myelinated structures in the CNS. (a) Top diagram representing areas of the forebrain imaged by reflectance, corpus callosum (CC) and cortical layer II-VI. Bottom diagram shows the specific areas of the corpus callosum (radial projections) and corpus callosum near the dorsolateral wall of the SVZ used for imaging. (b) Representative image from a 12- $\mu \mathrm{m}$ histological section of corpus callosum in naïve mice (left panel), showing reflectance in CC, near the lateral ventricle (LV). (b) Right panel: Shows projections between corpus callosum and cortical layers II-VI (arrow). Bar $=200 \mu \mathrm{m}$. (c) Representative CARS image of sebaceous glands of mouse skin; note the clear detail of cytoplasm and nucleus of sebaceous gland cells (arrow) and (inset); bar $=200 \mu \mathrm{m}$. (d) Diagram of areas of forebrain used to simultaneously image CARS and reflectance. (e) Side-by-side analysis of CARS and reflectance in forebrain. The CARS signal is increased in white matter compared to gray matter (cortex), and the signals from reflectance are present in both cortex and corpus callosum. ( $\mathrm{f}$ Visualization of developmental myelination of cerebellum. Top panel shows the area of forebrain that was examined, the middle panels show cerebellum at one week or seven weeks old; the reflectance signal was present at both ages. However, the CARS signal is absent in cerebellum at postnatal day 5 compared to week 7. (g) Quantification of the intensity of CARS signal during developmental myelination in the cerebellum, measured by 2.5-D LSM software at postnatal day 7 (left panel) compared to postnatal day 50 (right panel).

reflectance can be used to monitor axons in neurodegenerative diseases. In order to further verify that reflectance imaging visualizes axons, ${ }^{8,9}$ we used explants from Thy-1 yellow fluorescent protein (YFP) mice that express YFP in their axons and observed that the reflectance signal shows significant overlap with the YFP fluorescence, confirming the capabilities of reflectance to visualize axons as previously suggested ${ }^{8,9}$ [Fig. 3(b)]. The myelin sheaths normally surround axons in a spatially organized pattern. ${ }^{16}$ In order to study the relationship between myelin and axons, we performed simultaneous CARS and reflectance imaging of spinal cord explants of adult mice [Fig. 3(c)]. CARS and reflectance signals segregated independently without colocalization, allowing us to delineate individual axons surrounded by a myelin sheath as well as their individual profiles [Fig. 3(d)]. Using CARS measurements, we calculated a $g$ ratio of myelin to axons in the spinal cord of 0.65 , which is similar to a prior report $^{6}$ [Fig. 3(e)]. In addition, we could visualize other less compact structures in the axon-myelin interface, including the node of Ranvier, with an axon diameter of 10-12 $\mu \mathrm{m}$ in the internodal region and $2 \mu \mathrm{m}$ in the nodal region [Fig. 3(f), arrows]. Nodal constriction was confirmed by z-stack (data not shown). ${ }^{17}$ These findings suggest that CARS could be used to monitor not only primary demyelination but also axonal injury.

\subsection{Coherent Anti-Stokes Raman Scattering and Reflectance Microscopy Identify Demyelination and Axonal Alterations in Experimental Autoimmune Encephalomyelitis}

We have previously demonstrated that in the relapsing remitting model of EAE in the SJL strain of mice, there is a profound alteration of the cortical projecting neurons, especially obvious as they run through the corpus callosum. ${ }^{13}$ We used this model to examine the capacity of CARS and reflectance 


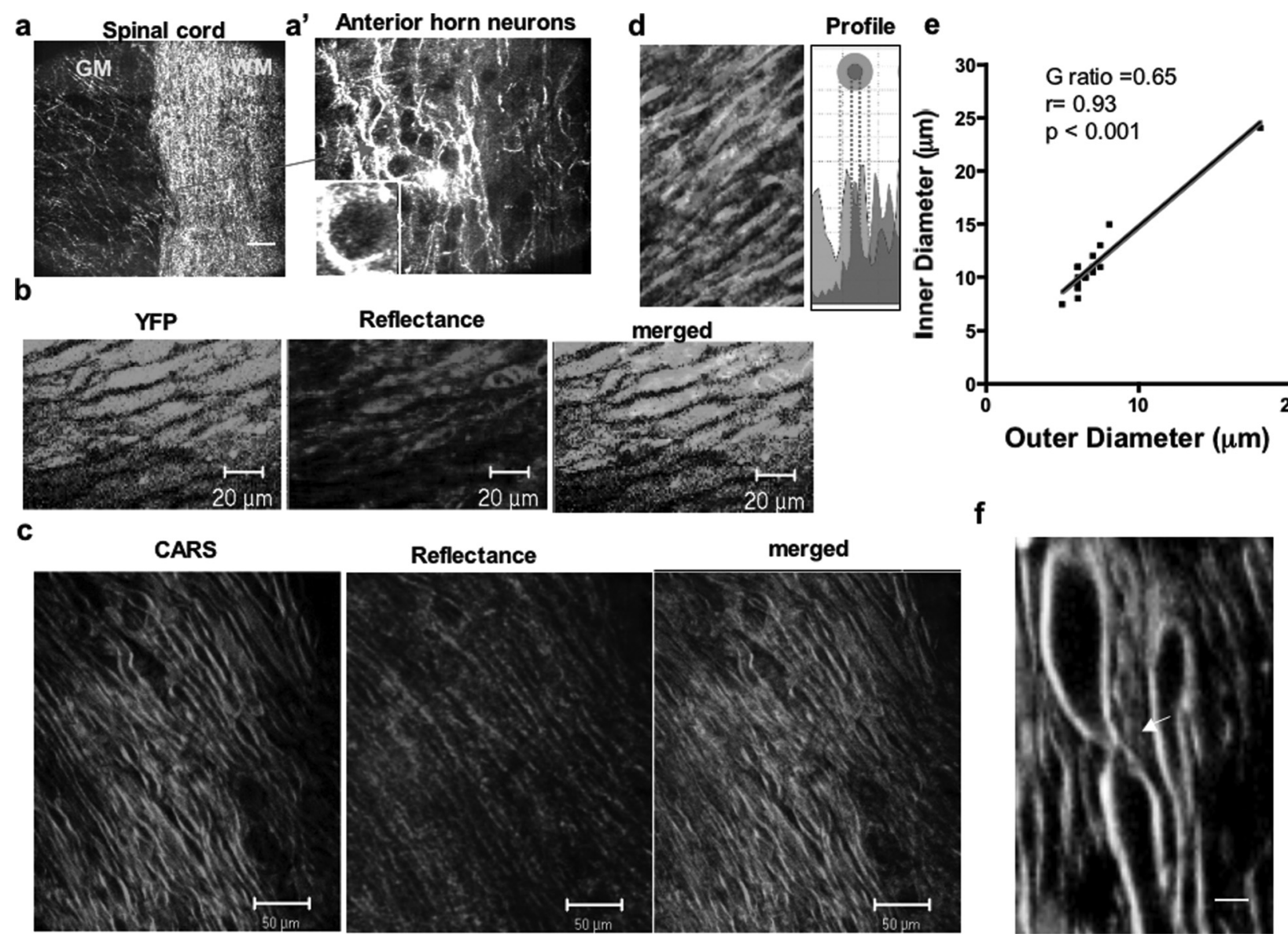

Fig. 3 CARS and reflectance demonstrate substructures of myelin. (a) Reflectance imaging of the spinal cord at the interface between white matter $(\mathrm{wm})$ and gray matter (gm), showing the increased reflectance signal in the white matter of the spinal cord. (a') Representative reflectance imaging of gray matter of the spinal cord (left panel) showing axon and dendrite arborizations with increased reflectance signal. The dark round profiles correspond to pyramidal neurons. Bar $=20 \mu \mathrm{m}$. (b) Representative imaging in the normal spinal cord of naïve mice showing colocalization of YFP and reflectance signals. (c) CARS imaging of normal myelin (green), reflectance (red), and merged picture. Note the alignment of the reflectance signal in myelin and axons. (d) Magnification of naïve spinal cord and Intensity profile of CARS and reflectance demonstrating segregation of the channels without overlap. (e) Linear correlation of inner diameter and outer diameter of multiple axons to determine the $g$ ratio detected by CARS imaging, $r=0.93$ with $p<0.001$. (f) Visualization of node of Ranvier by CARS (arrow); an axon diameter of 10-12 $\mu \mathrm{m}$ in the internodal region and $2 \mu \mathrm{m}$ in the nodal region. (Color online only.)

imaging modalities to simultaneously visualize the callosal and cortical pathology. ${ }^{13}$

SJL mice develop demyelination in the corpus callosum during EAE by day $25 \mathrm{dpi}$, seen as a profound rarefaction and loss of myelin shown by conventional histopathological staining with Luxol Fast Blue (LFB) [Fig. 4(d), bottom panel]. We used live tissue explants from the forebrain from such animals to seek axonal and myelin abnormalities by CARS and reflectance; we observed a robust reflectance signal in the corpus callosum and the projecting pathways [Fig. 4(b), left panel, arrows, and inset] in the control mice, whereas in animals with EAE, there was a significant reduction of the reflectance signal in the corpus callosum and the projecting axonal pathways in all animals examined [Fig. 4(b), right panel, arrows, and inset]. This was quantified by covariance of the pixel intensity in the reflectance images consistent with the alteration in axonal function that we have recently demonstrated by neuronal tracing studies ${ }^{13}$ [Fig. 4(c)]. To image the areas of demyelination, we acquired more than 20 individual optical sections of the corpus callosum in naïve and EAE mice. We found a diffuse reduction of the CARS signal in the corpus callosum of EAE mice as well as areas with severe decrease of CARS signal, correlating with the demyelination observed by LFB staining [Figs. 4(d)-4(f)]. ${ }^{13}$ These alterations are associated with a profound activation of microglia in the live explants as shown by time-lapse confocal microscopy ${ }^{13}$ and in ex vivo microdissected tissue stained for microglia (Fig. 5). Taken together, these data indicate that CARS and reflectance imaging modalities are useful to determine the occurrence of demyelination and axonal alterations in this model and further confirm our recently published observations of demyelination in the corpus callosum in these mice. ${ }^{13}$

\subsection{Coherent Anti-Stokes Raman Scattering and Reflectance Microscopy Identify Demyelination and Axonal Pathways in the Corticospinal Tracts in Experimental Autoimmune Encephalomyelitis Mice}

Spinal cord involvement in EAE is also severe and appears to be responsible for the majority of the motor symptoms, especially in mice of the C57BL6 strain immunized with MOG. Thus, 
a

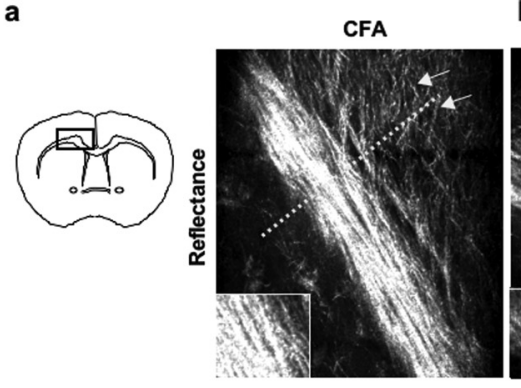
Luxol fast blue

d

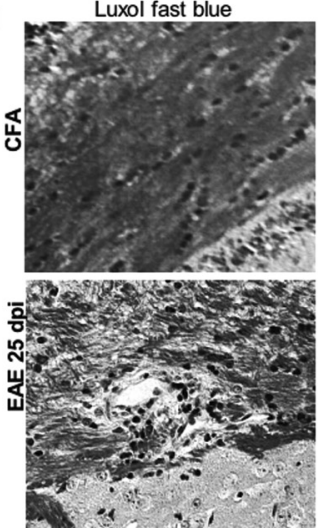

b
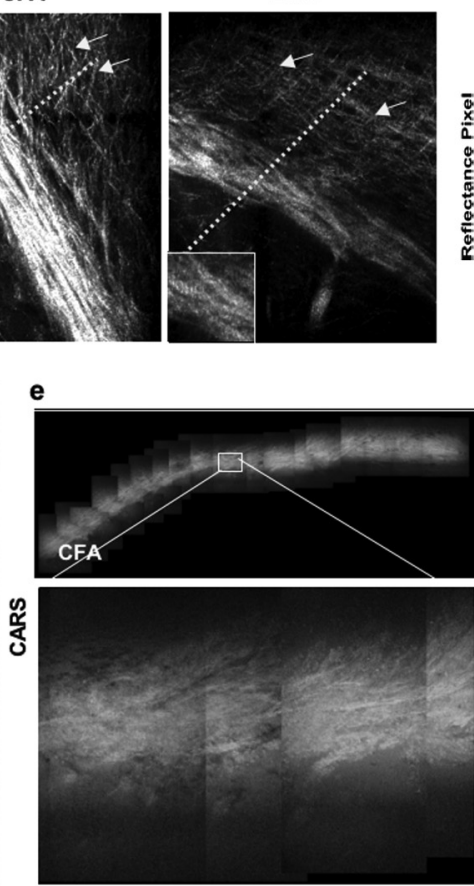

C

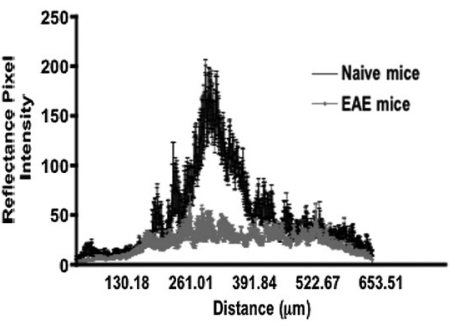

f
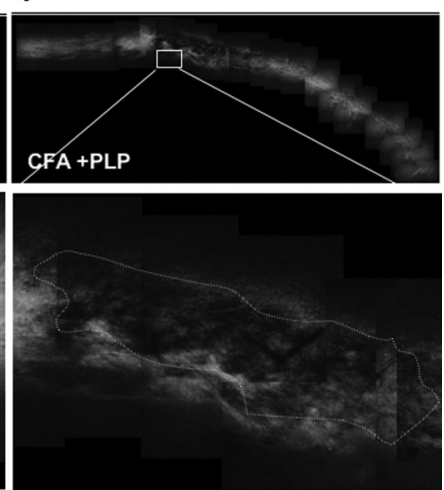

Fig. 4 Combination CARS and reflectance demonstrate axonal loss and demyelination in the forebrain. (a) Reflectance imaging of the corpus callosum of control mice immunized with CFA (left panel) and EAE mice (right panel). (b) Callosal projections of cortical layers VI (arrows) and the corpus callosum exhibit a decrease in the overall signal of reflectance that can be appreciated as well in the inset. (c) Quantification of the covariance of pixel intensity of reflectance signals in explants of corpus callosum, the covariance [dashed line in (a) and (b)] in the mean and standard error of the mean multiple lines of pixel intensity $(n=12)$. Control mice exhibit a typical peak in the corpus callosum and the projection pathways that are decreased in EAE (red). (d) LFB staining of EAE mice (bottom) at day 25 compared to control CFA (Top). (e) Large-scale reconstruction of map of individual CARS images $n=25$ from CFA animals, showing uniformity and increased CARS signal in the corpus callosum. (f) Visualization of large-scale map of corpus callosum in animals with EAE. A dark area of loss of CARS is indicative of frank demyelination. (Color online only).

we used CARS and reflectance imaging in these animals to study the spinal cord lesions. We mapped the spinal cord over a distance of $2 \mathrm{~mm}$ (Fig. 6(a)] and established the presence of areas with normal disposition of axons and myelin [Fig. 6(b)]. In diseased animals, boundaries of lesions within the spinal cord were clear [Fig. 6(f)]. The normal spinal cord shows parallel distribution of myelin sheath and axons with robust signal [Fig. 6(b)]. However, in the case of animals with EAE, we found a profound derangement of myelin sheath and a significant decrease of the reflectance signal, sometimes despite the apparent structural integrity of the myelin [Fig. 6(c)]. In EAE lesions, we found swelling of some myelin sheaths and round structures with myelin signals that appear to be conglomerates of degenerating myelin (arrows) [Fig. 6(d)]. Finally, in the normal-appearing white matter in EAE, there was tortuosity of myelinated axons, suggesting alterations of axons despite the preservation of myelin [Fig. 6(e)]. The myelin signal was quantified by covariance of pixel intensity showing areas of demyelination [Figs. 6(f) and 6(g)].

The hallmark of advanced axonal degeneration in EAE and MS is the presence of axonal ovoids, which represent alterations of axonal microtubules ${ }^{18}$ that precede Wallerian degeneration. ${ }^{16,19}$ Axonal ovoids demonstrated by staining with the nonphosphorylated form of neurofilament (SMI-32) SMI-32 are present in EAE but not in normal spinal cord [Figs. 6(h) and 6(i)]. We sought them also with the combination of CARS and reflectance, and easily identified ovoids in EAE and not in normal spinal cord as swelling of axons surrounded by myelin [Fig. 6(j)]. In order to verify that reflectance can be used to visualize abnormal axons, ${ }^{8,9}$ we used explants from Thy-1 YFP mice immunized with MOG 35-55 to induce EAE. In normal areas of the spinal cord of diseased mice, we observed the preservation of both YFP and reflectance signals [Fig. 6(k)], that was clearly abnormal in lesions that showed decrease YFP and reflectance signal [Fig. 6(1)]. Similarly, we confirmed that axonal ovoids visualized with reflectance [Fig. 6(j)], are clearly seen with YFP with similar colocalization with the reflectance signal [Fig. $6(\mathrm{~m})$ ]. These results confirmed the capabilities of reflectance to visualize normal and degenerating axons in EAE of YFP mice.

\subsection{Simultaneous Imaging of Myelin, Axons, and Green Fluorescent Protein in Chronic Experimental Autoimmune Encephalomyelitis}

We used animals that express GFP under the ubiquitin promoter (Ub-GFP) to visualize infiltrates of immune cells [Fig. 7(a)], the ubiquitin proteosome pathway being essential for activation of immune cells. ${ }^{20}$ Thus, activated $\mathrm{T}$ cells ${ }^{21}$ and microglia ${ }^{22}$ can be visualized by GFP expression and we hypothesized that the immune cell infiltrates will correlate with axonal and myelin alterations. We found aggregates of GFP-positive cells in proximity to extensive axonal alterations manifested by decreased reflectance signal [Figs. 7(b) and 7(c)]. Our previous results in the SJL mice showed that alterations of myelin and axons are associated with persistent activation of microglia, 


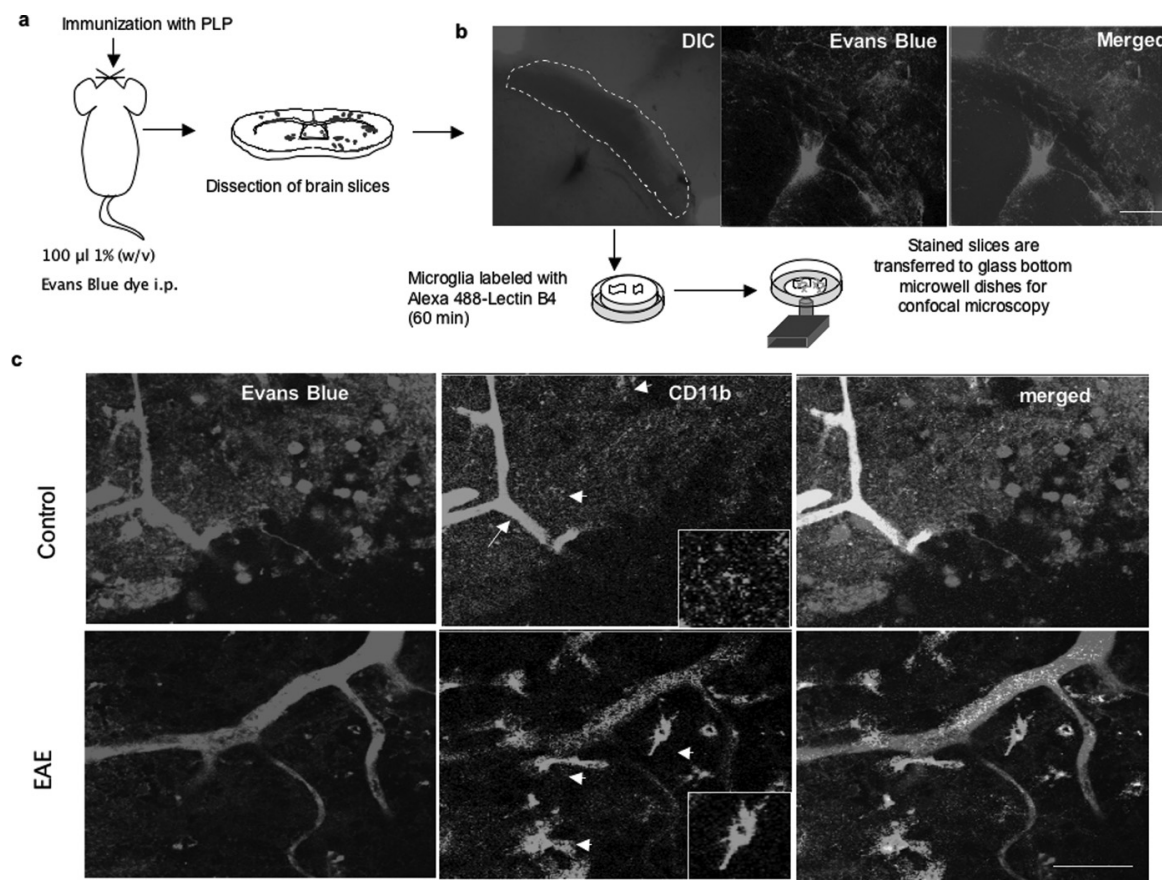

Fig. 5 Microdissection of forebrain tissue for ex vivo visualization of activated microglia in the SJL mice. (a) Diagram of methods used to stain microglia in brain slices with Alexa-488 IB4. (b) Representative confocal image of corpus callosum of EAE animals showing vessels and outlined area used for explants. (c) Representative image of the microglia in controls versus EAE animals in the corpus callosum of the SJL strain of mice; Evan blue stains vessels and lectin B4 stains microglia. In the control, there is strong staining in the perivascular microglia (arrow) and low in the parenchymal microglia (arrowhead). However in EAE, there is an increase in the staining (arrowhead), size, and numbers of parenchymal microglia cells compared to perivascular microglia cells (inset). These events are correlated with corpus callosum pathology as shown by CARS and reflectance imaging. Bars in $b=250 \mu \mathrm{m}$ and in $c=30 \mu \mathrm{m}$. (Color online only).

as shown previously, ${ }^{13}$ and confirmed here by CARS and reflectance imaging, combined with ex vivo staining of microglia with Lectin B4 (Figs. 4 and 5). In order to confirm these observations without the need of additional labeling or manipulations, we took advantage of the $\mathrm{CX}_{3} \mathrm{CR} 1-\mathrm{GFP}$ mice that express GFP in microglia, to study the association of microglia with the axonal and myelin alteration by CARS and reflectance imaging. Using a combination of modalities (CARS, reflectance, and confocal imaging), we found the typical distribution of axons and microglial cells that exhibit small body and long processes in the normal spinal cord [Figs. 7(d) and 7(e)]. This morphology changes dramatically in chronic EAE [Fig. 7(f)]. After immunization on day 30 and day 50, we observed abundant areas of activated microglia with shortened processes and increased expression of GFP [Figs. 7(d)—7(f)], correlating with a diffuse decrease in CARS signal and regional loss of reflectance [Fig. 7(g)]. These results demonstrate the visualization of EAE pathology with multimodal CARS, which showed alteration of axons and myelin closely associated with persistent microglial activation in the forebrain and the spinal cord in a mouse model of multiple sclerosis.

\section{Discussion}

Current imaging in models of multiple sclerosis, such as EAE, is limited to confocal microscopy in fixed tissue that shows neuronal and axonal alterations ${ }^{13,23}$ and live imaging of immune cell trafficking. ${ }^{24}$ Reports of techniques that allow the detection of demyelination in live tissue have been limited thus far. Nonetheless, CARS imaging has been used to visualize normal myelin and demyelination in a chemical model of demyelination ${ }^{6,7}$ and Wallerian degeneration following crush injuries. ${ }^{25}$ In MS and EAE, demyelination and axonal dysfunction are associated with inflammatory lesions. ${ }^{11}$ Thus, it would be advantageous to image alterations simultaneously in axons, myelin, and immune cells.

The mechanism of progression in MS is not known, but microglia have been associated with many of the hallmarks of progression, such as chronic inflammation, neurodegeneration, demyelination, axonal loss, and neuronal dysfunction. ${ }^{13,26}$ Methods that allow fast imaging of large areas with minimal or no staining or tissue processing, in addition to modalities that provide multiple complementary information about molecular and cellular aspects of the disease process, would be critical to $(i)$ advance our understanding of the disease, (ii) provide biomarkers for monitoring disease progress, and (iii) quantitatively evaluate therapeutic trials. In this paper, we demonstrate the use of a custom-built microscope that combines several nonlinear and linear modalities, including fluorescence and RCM and CARS microscopy. The combination of CARS, reflectance and GFP imaging allows fast ex vivo imaging of myelin, axons, and microglia in EAE with great anatomical precision in live tissue without the need for fixation and staining. These modalities can be used to monitor microglia-induced neurodegeneration in EAE. CARS microscopy measurements indicate a global decrease in myelination not seen before by any other technique, suggesting that subtle alterations in lipid content of myelin may occur even before frank demyelination correlated with axonal loss and microglial infiltrates ensues in localized CNS areas. In addition, in comparison with techniques used to 

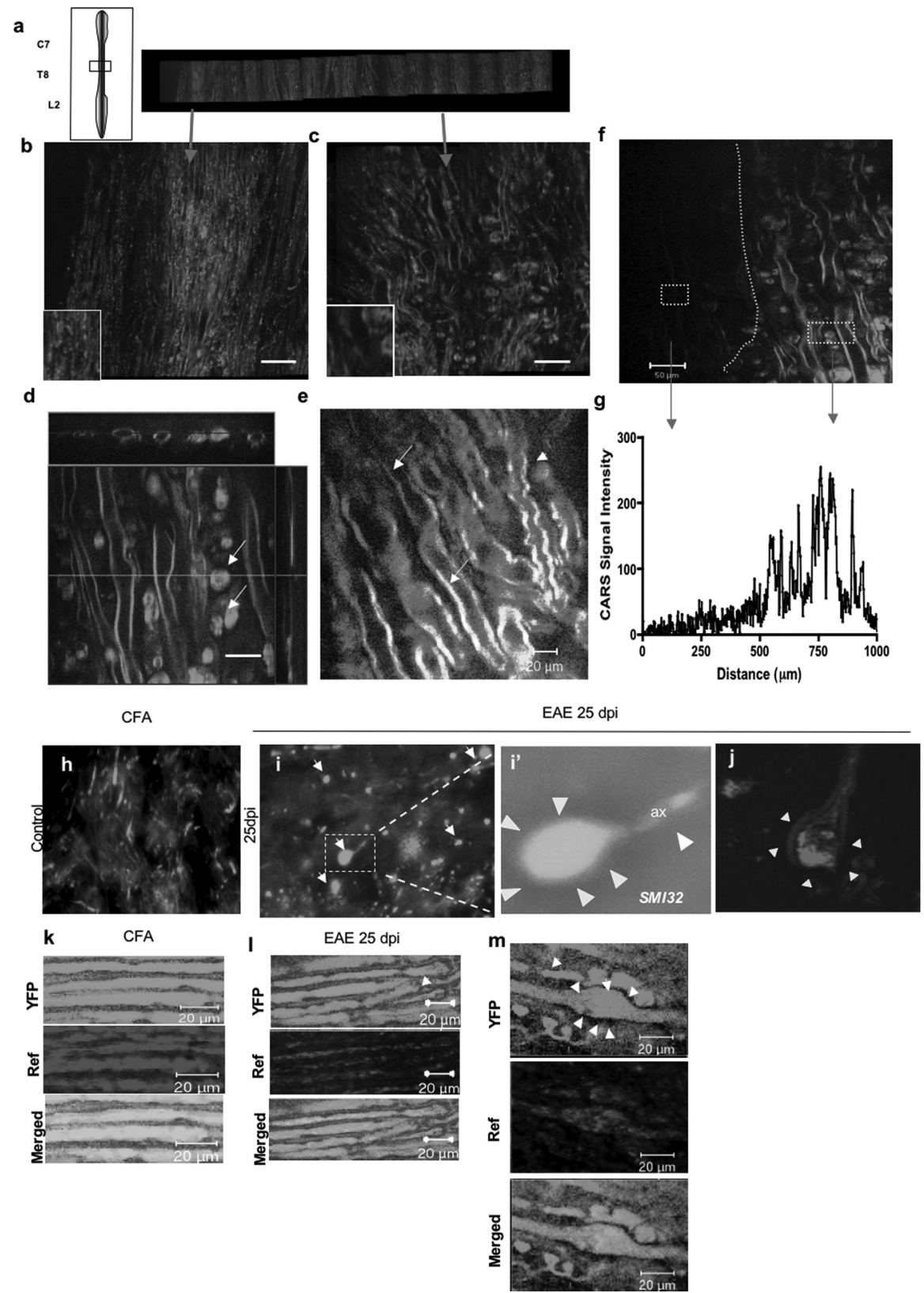

Fig. 6 Combination CARS and reflectance demonstrate axonal dysfunction and demyelination in the spinal cord. (a) Diagram and low-power map of a spinal cord slice of an EAE animal; several areas were highlighted for further analysis. (b) CARS imaging (green) combined with reflectance (red) allows one to observe the normal parallel distribution of myelinated axons in the spinal cord. (c) CARS signal during EAE at 30 dpi, with areas of complete loss of reflectance signal and areas of demyelination with dilatation of myelin sheaths. (d) Z-stack reconstruction of a dilated myelin sheath in EAE spinal cord, and accumulation of myelin debris (arrows). Bar $=20 \mu \mathrm{m}$. (e) Glow scale of CARS imaging in EAE spinal cord showing decrease in CARS signals in normal-appearing myelin (arrows) and tortuosity in myelin sheath (arrowhead). (f) CARS imaging showing border of demyelinating lesion in the spinal cord of EAE mice (dotted lines); the myelinated area show tortuosity of myelin sheath and myelin debris. (g) Quantification of CARS signal in demyelinating lesion, pixel intensity demonstrating total loss of CARS signal in the EAE lesion. (h) Confocal imaging of axonal SMI-32 staining in the normal spinal cord, showing typical distributions of axons, without abnormalities. (i) Confocal imaging of SMI-32 staining of EAE, showing an increase in formation of ovoids (arrows), transected axons (white arrowheads) that end in accumulation of microtubules (arrows), shown in inset $i^{\prime}$. (j) Visualization by CARS-reflectance imaging of axonal ovoids, with dilatation of axons surrounded by myelin (blue). (k) Visualization of YFP and reflectance signals in CFA-treated animals showing strong signal and colocalization of both imaging modalities. (I) Visualization of YFP and reflectance signals in diseased animals showing decrease in the YFP signal (arrow) with similar decrease in reflectance. (m) Axonal ovoid visualized with YFP (arrows), and reflectance. Bar $=20 \mu \mathrm{m}$. (Color online only).

measure demyelination, such as Luxol Fast Blue (LFB), the penetration and spatial resolution for CARS and confocal reflectance is deeper than regular immunohistochemistry and similar to confocal fluorescence, but provides more information than LFB, which requires multiple steps and nonliving tissue.
Furthermore, the combination of CARS and reflectance does not require any staining, which makes it very useful for rapid guidance and identification of pathology. The advantage is the ability to use on live tissue and observe cellular and molecular processes without the use of fixative and immunohistochemistry 


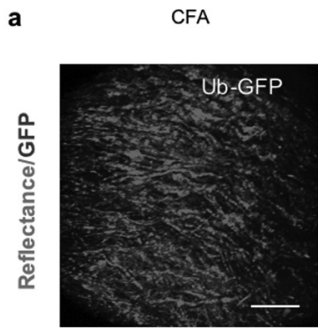

b

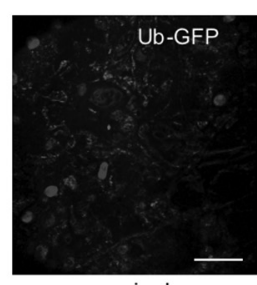

proximal
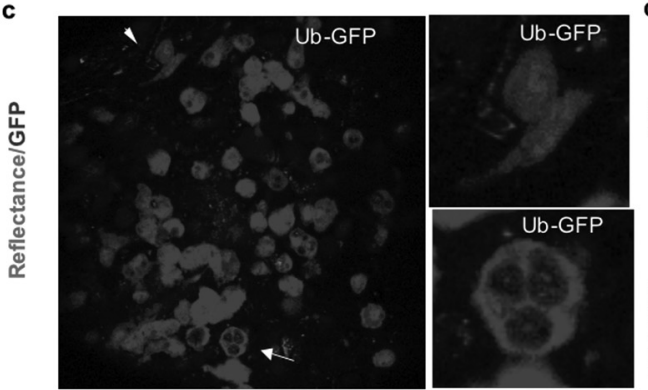

Reflectance

GFP
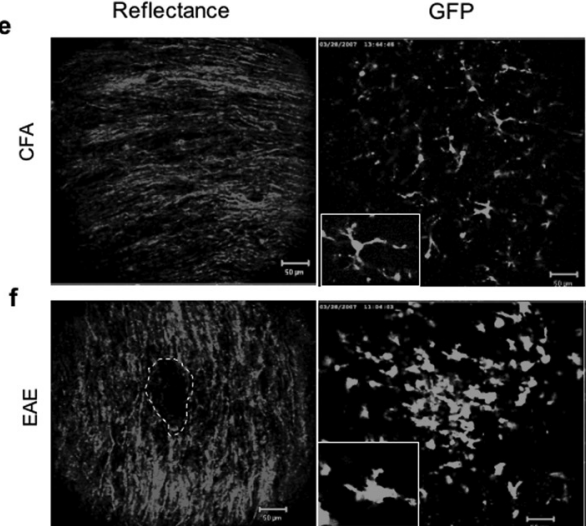

EAE 30dpi

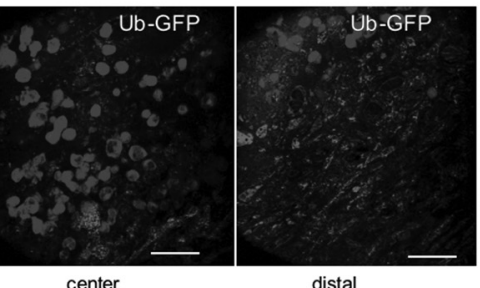

CX3CR1-GFP
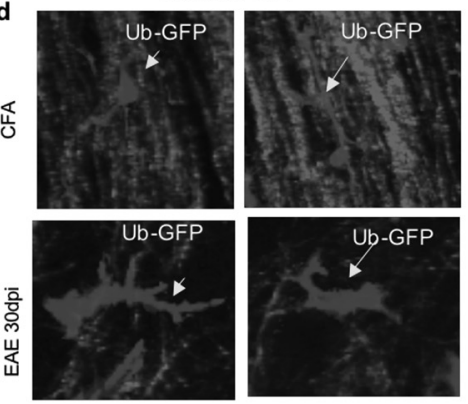

merged

CARS
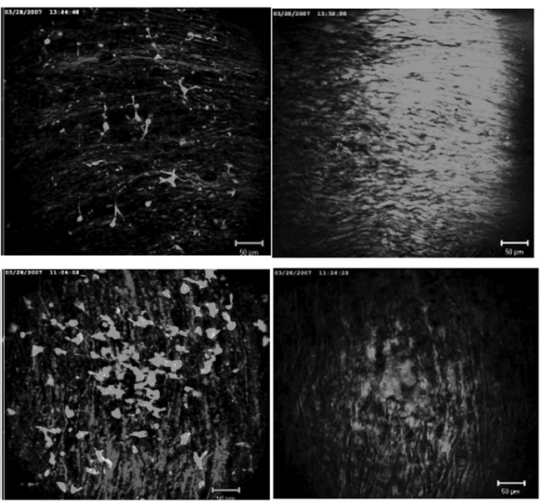

g

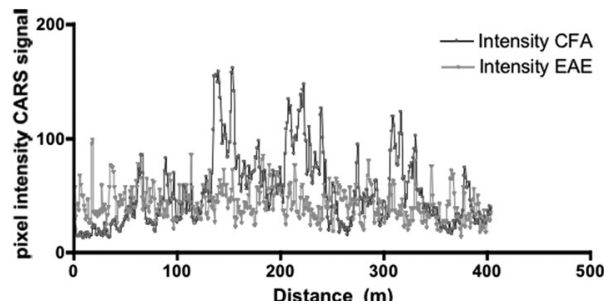

Fig. 7 Simultaneous imaging with CARS, reflectance and GFP. (a) Combination of reflectance and Ub-GFP in normal spinal cord that shows normal signal of axons and scattered GFP-positive cells. (b) Combination of reflectance and Ub-GFP in mice with EAE at day 30 . At the center of the lesion, there is an increased number of GFP-positive cells, with a decrease of the reflectance signal indicative of axonal damage. In areas distal to the lesion, there are fewer GFP cells and preservation of reflectance signal. (c) Areas of massive infiltrates showing increased number of GFP-positive cells that interact closely with axons and decreased reflectance signal (arrows). (d) Top panel shows 3-D projection of Z-stack of normal mouse spinal cord microglia and preserved reflectance signal; the microglial processes interact with surrounding axons (arrows in left top panel) or the cells extend long process parallel to the axonal axis (arrow in right top panel). Bottom panel shows 3-D projection of Z-stack of chronically activated microglial cells in EAE, with short processes and elongated cell body associated with decrease in reflectance signal (bottom left panel). The activated microglia also show changing morphology (bottom right panel). (e) Top panel shows reflectance (red), GFP-positive microglia (green), and merged and CARS imaging (green) of normal spinal cord explants. Note the discrete microglial cell body and fine process (bars $=50 \mu \mathrm{m})$. ( $\mathrm{f})$ Bottom panel demonstrate reflectance (red), GFP-positive microglia (green), and merged and CARS imaging (green) of spinal cord in animals at 45 dpi; note the microglial activation and changes in morphology and process, and activated microglial cells accumulated in nodules that correlate with alteration of axonal signals. The CARS signal shows a dramatic decrease in the same regions. (g) Quantification of pixel intensity of CARS imaging in normal spinal cord (black profile) areas devoid of microglia and areas full of microglial nodules in EAE (green profile). There is a significant decrease of the covariance of pixel intensity in EAE animals compared to naïve controls, $p<0.001$. (Color online only). 
or other staining methods. In regards to YFP, since reflectance is associated with functional integrity of microtubules, it could be used to monitor alterations in axons at very rapid time scales, providing a more physiological analysis of axons in real time.

In the mature uninjured CNS, microglial cells are highly branched or ramified as shown by GFP [Fig. 7(d)]. In response to injury, these so-called resting microglia becomes activated and undergo morphological and molecular changes that are proportional to the severity of the tissue injury. Activated microglia become deramified and develop an enlarged cell body with several short, thickened processes as shown in Fig. 7(d).

We have recently characterized the activation of microglia and its relationship with neuronal and axonal dysfunction, and observed that disease progression in the chronic relapsing remitting EAE model is related to diffuse and chronic microglial cell activation. ${ }^{13}$ In this report we demonstrate that the increased activity of microglia is associated with loss of myelin and axonal loss, shown by reflectance and CARS. Previously, we have demonstrated that microglia activation is associated with loss of synaptic proteins with the use of standard immunohistochemistry. ${ }^{13}$ Here we show without the use of staining, areas devoid of reflectance and decreased myelin, associated with (1) nodular presence of microglia, (2) change in morphology, and (3) expression of GFP in the CX3CR1 transgenic mice indicative of activation. Our present results extend these observations with use of novel visualization modalities based on chemical imaging of live tissue. We demonstrate that myelin lipid signals are abnormal very early and that, subsequently, axons become severely affected. Complete loss of axonal signal correlates with presence of highly activated microglial cells.

Our previous work showed abundant perivenular demyelination in the forebrain of SJL/J mice immunized with a synthetic peptide of myelin proteolipid protein (PLP) residues 139-151 and impaired axonal transport in the corpus callosum. ${ }^{13}$ Reflectance signals arise partly from microtubules, ${ }^{9}$ colocalize with axons, and are shown by YFP. They showed similar range of intensity as YFP, including decreased signal in axonal injury and accumulation in axonal ovoids, indicating alteration of the integrity of microtubules. ${ }^{18}$ Alteration in reflectance can provide functional data regarding axonal integrity and function. Further studies are needed to determine the kinetics of alteration of microtubules during axonal injury, and ovoid formation in relationship to the dynamics of changes in the reflectance signals in this model of demyelination.

We conclude that multimodal CARS microscopy has the potential to become a sensitive and important biomarker tool for study of mouse models of neurodegeneration, leading to improvement in our understanding of chronic progression and potential novel therapeutic strategies in mice and perhaps in humans.

\section{Materials and Methods}

\subsection{Animals}

Female SJL/J, C57BL6, C57BL6- $\mathrm{Ub}^{+}{ }^{+} g f p$, C57BL6$\mathrm{CX}_{3} \mathrm{CR} 1^{+/} g f p$ mice, C57BL6-Thy1.1 YFP mice were purchased from The Jackson Laboratory (Bar Harbor, Maine) and used at 6-8 weeks of age. Mice were housed four per cage and maintained on a 12-h light/dark cycle with food and water ad libitum. On arrival, mice were randomly assigned to exper- imental groups, followed by an acclimation period of at least three days prior to initiating experiments.

\subsection{Experimental Autoimmune Encephalomyelitis Induction}

$\mathrm{SJL} / \mathrm{J}$ mice were immunized subcutaneously in two sites (left and right flank) on day 0 with $150 \mu \mathrm{g}$ of PLP139-151 emulsified in complete Freund's adjuvant containing $200 \mu \mathrm{g}$ Mycobacterium tuberculosis. Mice were injected intraperitoneally with $200 \mathrm{ng}$ pertussis toxin on the day of immunization and two days later. $^{13}$

MOG-induced EAE in C57BL6, C57BL6-CX ${ }_{3} \mathrm{CR} 1^{+/} g f p$, and Thy1.1-YFP mice were induced as previously described. ${ }^{27}$ Briefly, C57BL/6J mice were immunized subcutaneously with $200 \mathrm{ng}$ of MOG peptide in phosphate buffered saline (PBS) and complete Freund's adjuvant containing $0.4 \mathrm{mg}$ M. tuberculosis and intraperitoneally injected with $200 \mathrm{ng}$ pertussis toxin on the day of immunization and two days later. Control mice were immunized with complete Freund's adjuvant followed by pertussis toxin. Mice were scored daily for neurological signs as follows: 0 , no disease; 1 , loss of tail tone; 2 , hind-limb weakness; 3 , hind-limb paralysis; 4, scale 3 plus forelimb weakness; and 5, moribund. For immunohistology, animals were sacrificed, perfused with $4 \%$ paraformaldehyde in PBS; tissues were harvested and postfixed, dehydrated with sucrose $30 \%$, and placed in OCT and stored at $-80^{\circ} \mathrm{C}$ until used. ${ }^{13}$

\subsection{Acute Forebrain and Spinal Cord Slices}

Transverse spinal cord slices $250-400 \mu \mathrm{m}$ thick were prepared from (6-8)-week-old mice and were incubated at $34^{\circ} \mathrm{C}$ in artificial cerebrospinal fluid (ACSF) containing $118 \mathrm{mM} \mathrm{NaCl}, 3 \mathrm{mM}$ $\mathrm{KCL}, 1 \mathrm{mM} \mathrm{KH} \mathrm{PO}_{4}, 1 \mathrm{mM} \mathrm{MgSO}{ }_{4}, 20 \mathrm{mM}$ glucose, $1.5 \mathrm{mM}$ $\mathrm{CaCl}_{2}$, and $25 \mathrm{mM} \mathrm{NaHCO}$. The ACSF was oxygenated with $95 \% \mathrm{O}_{2}$ and $5 \% \mathrm{CO}_{2}$. Imaging was performed in ACSF-filled glass-bottom culture dishes (World Precision Instruments, Sarasota, Florida) at room temperature or $37^{\circ} \mathrm{C}$ under a temperature controller.

\subsection{Multimodal Coherent Anti-Stokes Raman Scattering Video-Rate Microscope}

Reflectance and CARS microscopy were performed simultaneously. The microscope and laser sources were previously described. ${ }^{15}$ Briefly, we achieved CARS video-rate imaging of live tissues by selecting a light source and microscope design that produce strong vibrational signals under benign excitation conditions. Modifications ${ }^{14}$ from a previously described design ${ }^{15}$ include a reflectance confocal channel, an improved filter stack before the nondescanned PMT (Chroma, 650-nm bandpass with over $90 \%$ transmission), an electronic limiter for analog thresholding of the CARS signal from the PMT, and a custom-built multichannel acquisition system with averaging, photon-counting, and live streaming capabilities. After losses through the scanning system, the dichroic (800-nm shortpass) and the 63X objective, a total of 50-100 $\mathrm{mW}$ (pump and Stokes beams) is incident on the sample. The contrast obtained with coherent anti-Stokes microscopy arises because the frequency difference of the pump and Stokes beam is chosen to resonantly 
excite the vibrational resonance $\mathrm{CH}_{2}$ stretch mode predominantly localized in lipids. The CARS microscope consists of two synchronized laser sources with a custom-built video-rate laser-scanning system. A 10-W, 7-ps Nd:Vanadate pulsed laser (picoTRAIN, High Q Laser) operating at $1.064 \mu \mathrm{m}$ (the CARS Stokes wavelength) is sent to a half-waveplate and a polarizing beam splitter, after which $\sim 20 \%$ of the power is injected into an optical parametric oscillator [(OPO), Levante, APE]. The synchronously pumped OPO generates $\sim 400 \mathrm{~mW}$ at a wavelength of $816 \mathrm{~nm}$ (the CARS pump wavelength). The remaining $80 \%$ of the $1.064-\mu \mathrm{m}$ beam is sent to a neutral density wheel and a 1:1 telescope used to mode match the wavefront of the OPO. Both beams are recombined with a dichroic mirror (CVI laser) and are sent to a rotating 36-face polygonal mirror operating at 500 rotations per second, yielding a $16-\mathrm{kHz}$ line refresh rate. The pivot point is imaged through a 4:7.5 telescope onto a galvanometer mirror that provides scanning in the vertical direction at $30 \mathrm{~Hz}$. Before acquisition at the PMT, signal limiting is performed to restrict the amplitude to $1.5 \mathrm{~V}$. The photomultiplier tubes are used in analog mode. In addition, we have custom built a thresholder for counting photons. This strategy allows us to use the photon counting mode by simply running at a lower dynode voltage. This way the dynamic range can be preserved when needed and allow us to count photons when flux is low.

\subsection{Postacquisition Image Processing}

All videos and digital data were processed with custom-made software. Thresholding is performed to normalize the photon signals and perform photon counting, therefore increasing contrast in low signal regimes. In addition, raw data were imported as in the LSM 510 software version 2.8 to be analyzed for colocalization, pixel intensity covariance, and 3-D reconstructions as previously described. ${ }^{28-31}$

\subsection{Tissue Processing}

At the selected end points, mice were deeply anesthetized in a $\mathrm{CO}_{2}$ chamber and transcardially perfused with cold PBS followed by $4 \%$ cold paraformaldehyde solution (PFA) in PBS. Brains were removed and postfixed in PFA for $48 \mathrm{~h}$.

\subsection{Histology}

Six-micrometer-thick paraffin sections of the forebrain were cut and stained with hematoxylin and eosin to assess cellular infiltration, LFB for myelin or Bielschowsky silver stain for axons. A number of inflammatory foci, identified as perivascular clusters containing at least 20 mononuclear cells, were counted in the cerebral cortex, corpus callosum, striatum, and septum. Areas with loss of LFB parenchymal staining (indicating demyelination) or loss of Bielschowsky staining (indicating axonal loss) were compared to similar areas in age-matched controls.

\subsection{Immunohistochemistry}

Brains and spinal cords were placed in $30 \%$ sucrose for at least $24 \mathrm{~h}$ for cryoprotection. Brains were divided into four serial blocks (olfactory bulb, subventricular zone, hippocampus, and pons/cerebellum) and spinal cords were divided into two blocks (cervical and lumbar regions). These tissue blocks were frozen in cryoprotective solution (tissue-freezing medium) at $-80^{\circ} \mathrm{C}$. The tissue was cut into floating $40-\mu \mathrm{m}$ sections on a freezing microtome. Floating sections were blocked with $8 \%$ horse serum for $1 \mathrm{~h}$ and incubated overnight with rat antibody against CD11b (1:20, BD Biosciences, San Jose, California) and mouse anti smi-32 antibody (1:250 Sternberger Monoclonals Inc., Lutherville, Maryland). Sections were rinsed and incubated for $1 \mathrm{~h}$ with the appropriate Alexa Fluor 488 and 594 secondary antibodies (1:500, Molecular Probes, Eugene, Oregon). Negative control sections for each animal received identical preparation for immunostaining, except that primary antibodies were omitted.

\subsection{Confocal Analysis}

Cells labeled with CD11b were reconstructed with a confocal microscope (LSM 510 Laser Scanning Microscope and LSM 3-D analysis software, Linux, Ogdensburg, New York), 63X objective, by taking a series of images through the $z$-axis in the region of interest. These 3-D reconstructions allowed analysis of 2-D image planes for quantification of morphological changes of microglia at different time points.

\subsection{Tissue Slice Preparation for Ex Vivo Microglia Staining and Confocal Analysis}

Ex vivo staining of microdissected brain was done using a previously described protocol. ${ }^{13}$ Briefly, tissue of interest from corpus callosum was dissected out and sliced at a thickness of $200 \mu \mathrm{m}$ with a McIlwain tissue slicer (Brinkmann, El Cajon, California). The slices were then placed on a coverslip precoated with poly-L-lysine. To promote attachment of the tissues, a hydrogel (BD Bioscience, San Diego, California) solution was added on top of the tissue, and the Petri dishes with coverslips were placed in an incubator for $30 \mathrm{~min}$ to promote gelation. Some mice were injected intraperitoneally with $100 \mu \mathrm{l}$ of $1 \%(\mathrm{w} / \mathrm{v})$ Evans Blue dyel we removed brains 1-h later and examined 1-mm slices for leakage of Evans Blue dye to label all major blood vessels in the brain and localize lesions in the corpus callosum that contained activated microglia. Fluorescent staining of microglia in living brain tissue slices was performed with Alexa $488 \mathrm{IB}_{4}$ (Molecular Probes) in a six-well plate with 30-mm culture-plate inserts (Millipore Corporation, Billerica, Massachusetts) by adding a stock solution to the culture medium at a final concentration of $5 \mu \mathrm{g} / \mathrm{ml}$ for $60 \mathrm{~min}$.

\subsection{Statistical Analysis}

Statistical analysis was performed with one-way analysis of variance with Turkey's Multiple Comparison Test. A $p$ value of $<0.05$ was considered significant.

\section{Acknowledgments}

This study was funded through the following: Grant Nos. RG2988 and RG35041 from NMSS, Grant Nos. AI043496 and AI058680 from NIAID to S. J. K.; Grant No. EB000664 from NIBIB to C. P. L.; NSERC Canada Research Chairs to D. C.; 
Imitola et al.: Multimodal coherent anti-Stokes Raman scattering microscopy reveals microglia-associated myelin...

Grant No. KO8 NS 047669-01 from NINDS to T. C.; NMSS Pilot Project No. 1239 to J. I.; and a faculty PhD scholarship from University of Southern Denmark and a grant from Hørslev Fonden in Denmark to S. R.. The authors thank Dr. Bing $\mathrm{Zhu}, \mathrm{MD}, \mathrm{PhD}$, for critical reading of the manuscript and suggestions.

\section{References}

1. H. S. David M, and B. Altevogt, Neuroscience Biomarkers and BIosignatures: Covering Technologies, Emerging Partnerships-Workshop Summary, National Academies Press, Washington, D.C. (2008).

2. D. Davalos, J. Grutzendler, G. Yang, J. V. Kim, Y. Zuo, S. Jung, D. R. Littman, M. L. Dustin, and W. B. Gan, "ATP mediates rapid microglial response to local brain injury in vivo," Nat. Neurosci. 8(6), 752-758 (2005)

3. A. Nimmerjahn, F. Kirchhoff, and F. Helmchen, "Resting microglial cells are highly dynamic surveillants of brain parenchyma in vivo," Science 308(5726), 1314-1318 (2005).

4. M. Nuriya, J. Jiang, B. Nemet, K. B. Eisenthal, and R. Yuste, "Imaging membrane potential in dendritic spines," Proc. Natl. Acad. Sci. USA 103(3), 786-790 (2006).

5. R. D. Roorda, T. M. Hohl, R. Toledo-Crow, and G. Miesenbock, "Video-rate nonlinear microscopy of neuronal membrane dynamics with genetically encoded probes," J. Neurophysiol. 92(1), 609-621 (2004).

6. H. Wang, Y. Fu, P. Zickmund, R. Shi, and J. X. Cheng, "Coherent anti-stokes Raman scattering imaging of axonal myelin in live spinal tissues," Biophys. J. 89(1), 581-591 (2005).

7. Y. Fu, H. Wang, T. B. Huff, R. Shi, and J. X. Cheng, "Coherent anti-Stokes Raman scattering imaging of myelin degradation reveals a calcium-dependent pathway in lyso-PtdCho-induced demyelination," J. Neurosci. Res. 85(13), 2870-2881 (2007).

8. X. R. Huang, R. W. Knighton, and L. N. Cavuoto, "Microtubule contribution to the reflectance of the retinal nerve fiber layer," Invest. Ophthalmol. Vis. Sci. 47(12), 5363-5367 (2006).

9. R. W. Knighton, X. Huang, and Q. Zhou, "Microtubule contribution to the reflectance of the retinal nerve fiber layer," Invest. Ophthalmol. Vis. Sci. 39(1), 189-193 (1998).

10. M. A. Middelkamp-Hup, H. Y. Park, J. Lee, B. A. Gilchrest, and S. Gonzalez, "Detection of UV-induced pigmentary and epidermal changes over time using in vivo reflectance confocal microscopy," $J$. Invest. Dermatol. 126(2), 402-407 (2006).

11. J. Imitola, T. Chitnis, and S. J. Khoury, "Insights into the molecular pathogenesis of progression in multiple sclerosis: potential implications for future therapies," Arch. Neurol. 63(1), 25-33 (2006).

12. B. Zhu, I. Guleria, A. Khosroshahi, T. Chitnis, J. Imitola, M. Azuma, H. Yagita, M. H. Sayegh, and S. J. Khoury, "Differential role of programmed death-ligand 1 [corrected] and programmed death-ligand 2 [corrected] in regulating the susceptibility and chronic progression of experimental autoimmune encephalomyelitis," J. Immunol. 176(6), 3480-3489 (2006).

13. S. Rasmussen, Y. Wang, P. Kivisakk, R. T. Bronson, M. Meyer, J. Imitola, and S. J. Khoury, "Persistent activation of microglia is associated with neuronal dysfunction of callosal projecting pathways and multiple sclerosis-like lesions in relapsing-remitting experimental autoimmune encephalomyelitis," Brain 130(Pt 11), 2816-2829 (2007).

14. S. J. Veilleux I, D. Biss, D. Cote, and C. P. Lin, "In vivo cell tracking with multimodal video rate microscopy," Biophotonics 14(1), 10-18 (2008).

15. C. L. Evans, E. O. Potma, M. Puoris'haag, D. Cote, C. P. Lin, and X. S. Xie, "Chemical imaging of tissue in vivo with video-rate coherent anti-Stokes Raman scattering microscopy," Proc. Natl. Acad. Sci. USA 102(46), 16807-16812 (2005)

16. B. Kornek, M. K. Storch, J. Bauer, A. Djamshidian, R. Weissert, E. Wallstroem, A. Stefferl, F. Zimprich, T. Olsson, C. Linington, M. Schmidbauer, and H. Lassmann, "Distribution of a calcium channel subunit in dystrophic axons in multiple sclerosis and experimental autoimmune encephalomyelitis," Brain 124(Pt 6), 1114-1124 (2001).
17. R. J. Reynolds and J. W. Heath, "Patterns of morphological variation within myelin internodes of normal peripheral nerve: quantitative analysis by confocal microscopy," J. Anat. 187(Pt 2), 369-378 (1995).

18. A. Erturk, F. Hellal, J. Enes, and F. Bradke, "Disorganized microtubules underlie the formation of retraction bulbs and the failure of axonal regeneration," J. Neurosci. 27(34), 9169-9180 (2007).

19. B. Kornek, M. K. Storch, R. Weissert, E. Wallstroem, A. Stefferl, T. Olsson, C. Linington, M. Schmidbauer, and H. Lassmann, "Multiple sclerosis and chronic autoimmune encephalomyelitis: a comparative quantitative study of axonal injury in active, inactive, and remyelinated lesions," Am. J. Pathol. 157(1), 267-276 (2000).

20. S. Menon, H. Chi, H. Zhang, X. W. Deng, R. A. Flavell, and N. Wei, "COP9 signalosome subunit 8 is essential for peripheral $\mathrm{T}$ cell homeostasis and antigen receptor-induced entry into the cell cycle from quiescence," Nat. Immunol. 8(11), 1236-1245 (2007).

21. L. P. Shriver and B. N. Dittel, "T-cell-mediated disruption of the neuronal microtubule network: correlation with early reversible axonal dysfunction in acute experimental autoimmune encephalomyelitis," Am. $J$. Pathol. 169(3), 999-1011 (2006).

22. H. Takeuchi, T. Mizuno, G. Zhang, J. Wang, J. Kawanokuchi, R. Kuno, and A. Suzumura, "Neuritic beading induced by activated microglia is an early feature of neuronal dysfunction toward neuronal death by inhibition of mitochondrial respiration and axonal transport," J. Biol. Chem. 280(11), 10444-10454 (2005).

23. P. G. Bannerman, A. Hahn, S. Ramirez, M. Morley, C. Bonnemann, S. Yu, G. X. Zhang, A. Rostami, and D. Pleasure, "Motor neuron pathology in experimental autoimmune encephalomyelitis: studies in THY1-YFP transgenic mice," Brain 128(Pt 8), 1877-1886 (2005).

24. N. Kawakami, U. V. Nagerl, F. Odoardi, T. Bonhoeffer, H. Wekerle, and A. Flugel, "Live imaging of effector cell trafficking and autoantigen recognition within the unfolding autoimmune encephalomyelitis lesion," J. Exp. Med. 201(11), 1805-1814 (2005).

25. F. P. Henry, D. Cote, M. A. Randolph, E. A. Rust, R. W. Redmond, I. E. Kochevar, C. P. Lin, and J. M. Winograd, "Real-time in vivo assessment of the nerve microenvironment with coherent anti-Stokes Raman scattering microscopy," Plast. Reconstr. Surg. 123(2 Suppl), 123S-130S (2009).

26. A. Kutzelnigg, C. F. Lucchinetti, C. Stadelmann, W. Bruck, H. Rauschka, M. Bergmann, M. Schmidbauer, J. E. Parisi, and H. Lassmann, "Cortical demyelination and diffuse white matter injury in multiple sclerosis," Brain 128(Pt 11), 2705-2712 (2005).

27. A. D. Salama, T. Chitnis, J. Imitola, M. J. Ansari, H. Akiba, F. Tushima, M. Azuma, H. Yagita, M. H. Sayegh, and S. J. Khoury, "Critical role of the programmed death-1 (PD-1) pathway in regulation of experimental autoimmune encephalomyelitis," J. Exp. Med. 198(1), 71-78 (2003).

28. J. Imitola, K. Raddassi, K. I. Park, F. J. Mueller, M. Nieto, Y. D. Teng, D. Frenkel, J. Li, R. L. Sidman, C. A. Walsh, E. Y. Snyder, and S. J. Khoury, "Directed migration of neural stem cells to sites of CNS injury by the stromal cell-derived factor $1 \mathrm{alpha/CXC} \mathrm{chemokine}$ receptor 4 pathway," Proc. Natl. Acad. Sci. USA 101(52), 18117-18122 (2004).

29. A. Monsonego, J. Imitola, S. Petrovic, V. Zota, A. Nemirovsky, R. Baron, Y. Fisher, T. Owens, and H. L. Weiner, "Abeta-induced meningoencephalitis is IFN-gamma-dependent and is associated with T cell-dependent clearance of Abeta in a mouse model of Alzheimer's disease," Proc. Natl. Acad. Sci. USA 103(13), 5048-5053 (2006).

30. S. Xiao, N. Najafian, J. Reddy, M. Albin, C. Zhu, E. Jensen, J. Imitola, T. Korn, A. C. Anderson, Z. Zhang, C. Gutierrez, T. Moll, R. A. Sobel, D. T. Umetsu, H. Yagita, H. Akiba, T. Strom, M. H. Sayegh, R. H. DeKruyff, S. J. Khoury, and V. K. Kuchroo, "Differential engagement of Tim-1 during activation can positively or negatively costimulate T cell expansion and effector function," J. Exp. Med. 204(7), 1691-1702 (2007).

31. C. Zhu, A. C. Anderson, A. Schubart, H. Xiong, J. Imitola, S. J. Khoury, X. X. Zheng, T. B. Strom, and V. K. Kuchroo, "The Tim-3 ligand galectin- 9 negatively regulates T helper type 1 immunity," Nat. Immunol. 6(12), 1245-1252 (2005). 